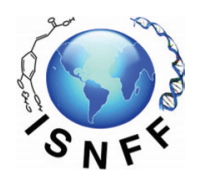

\title{
Launch of Journal of Food Bioactives (JFB)
}

\author{
Fereidoon Shahidi
}

The relationship between food, nutrition and health has led to the development of nutraceuticals and functional foods. Demonstrated physiological benefit of such commodities, whether food, extracts, phytochemicals and bioactives, natural health products as well as herbals and traditional medicine is gradually filling the existing gap between food and medicine which has existed for centuries. The physiological benefits starts with in-vitro investigations followed by cell line studies, pre-clinical animal testing and eventually human clinical trials. The bioactive components of food and non-food items need to be identified first and for this, their extraction from the matrix is needed. Different methods, including green processes are often practiced and these, together with nutrigenomics, proteomics, lipidomics and foodomics have led to impressive developments that may lead to individualized nutrition. Of course delivery of food bioactives, if not in the food itself, necessitates use of sophisticated technologies such as nanotechnology and stabilization during processing and storage, in order to reach physiologically relevant levels. The science generated not only promotes health but also reduces health care costs that are burdening individuals and /or governments. The International Society for Nutraceuticals and Functional Foods (ISNFF) which began its activity in this ever-growing field in 2008 is proud to start another initiative by launching JFB to complement existing efforts in the field and for a society-based journal which is supported by dedicated internationally reputed scientists and relevant associates. I am impressed with the support we have already received from the scientific community in this regard. An internationally recognized editorial board ensures that only high quality manuscripts are included in our new journal. I encourage you to submit your novel findings and research results in the form of full papers, review articles, short communications, perspectives and opinion pieces to our journal.

Fereidoon Shahidi

Editor-in-Chief Department of Biochemistry Memorial University of Newfoundland

St. John's, NL, Canada AlB 3X9 Tel.: +1 709864 8552; fax: +1 7098642422

E-mail address: fshahidi@mun.ca Available online March 2018 